\title{
MULTIPLE HARMONIC PLASMA EMISSION
}

\author{
Tongnyeol Rhee ${ }^{1}$, Chang-Mo Ryu ${ }^{1}$, Minho Woo ${ }^{1}$, Helen H. KaAng ${ }^{1}$, Sumin Yi ${ }^{1}$, and Peter H. Yoon ${ }^{1,2,3}$ \\ ${ }^{1}$ Department of Physics, POSTECH, Pohang, South Korea \\ Received 2008 September 19; accepted 2008 December 23; published 2009 March 17
}

\begin{abstract}
Electromagnetic radiation at the plasma frequency and/or its second harmonic, the so-called plasma emission, is widely accepted as the fundamental process responsible for solar type II and III radio bursts. There have also been occasional observations of higher-harmonic plasma emissions in the solar-terrestrial environment. This paper presents the first demonstration of multiple harmonic emission by means of twodimensional electromagnetic particle-in-cell simulation. This finding indicates that under certain circumstances the traditional mechanism of fundamental-harmonic pair emission might also be accompanied by higher-harmonic components. Consequently, the present findings are highly relevant to in situ observations of third- and/or higher-harmonic plasma emission in astrophysical and solar-terrestrial environments.
\end{abstract}

Key words: Sun: radio radiation

Online-only material: color figures

\section{INTRODUCTION}

The emission of electromagnetic (EM) radiation at the fundamental (F) plasma frequency and/or its second harmonic $(2 \mathrm{H})$, the so-called plasma emission, is well known. Classic examples are the solar type II and III radio bursts (Goldman 1983; Melrose 1985; Robinson \& Cairns 1998a, 1998b, 1998c). Laboratory beam-plasma interaction experiments also demonstrate plasma emission or equivalent phenomenon (Hutchinson et al. 1978; Benford et al. 1980; Whelan \& Stenzel 1985a, 1985b; Intrator et al. 1984). One of the most commonly accepted mechanisms for the $2 \mathrm{H} \mathrm{EM}$ emission involves the merging of two oppositely traveling Langmuir waves, compactly expressed as $\mathrm{L}+\mathrm{L}^{\prime} \rightarrow 2 \mathrm{H}$, where $\mathrm{L}$ stands for the primary Langmuir wave traveling along the electron beam, while $\mathrm{L}^{\prime}$ represents the backscattered Langmuir wave traveling opposite to L. Here, $2 \mathrm{H}$ stands for the $2 \mathrm{H} \mathrm{EM}$ wave. For the emission at the fundamental, the suggested radiation mechanism is either the decay of Langmuir waves into transverse and ion-sound (S) waves, $\mathrm{L} \rightarrow$ $\mathrm{S}+\mathrm{F}$, or the induced scattering of Langmuir wave into $\mathrm{F}$ radiation mediated by thermal ions (i), $\mathrm{L}+\mathrm{i} \rightarrow \mathrm{F}$ (Melrose 1985). Which of the two competing mechanisms, namely, the decay or scattering, is dominant for the fundamental emission has not been settled conclusively, however. At any rate, under the customary theory that involves either the three-wave merging or induced scattering, it is difficult to account for the radiation emission at third harmonic (3H) or higher (Cairns 1987).

Higher-harmonic emissions accompanying $\mathrm{F}$ and $2 \mathrm{H}$ emission have been reported in space environment. $3 \mathrm{H}$ emissions have been reported in type II bursts (Kliem et al. 1992; Zlotnik et al. 1998) and type III bursts (Takakura \& Yousef 1974; Benz 1973). Multiple harmonic emission up to fifth harmonic $(5 \mathrm{H})$ in the Earth's foreshock environment has been reported (Cairns 1986). Various theories have been put forth in order to explain the higher-harmonic emission. The coalescence of three Langmuir waves, $\mathrm{L}+\mathrm{L}^{\prime}+\mathrm{L}^{\prime \prime} \rightarrow 3 \mathrm{H}$, was suggested for $3 \mathrm{H}$ emission (Kliem et al. 1992), but such a theory obviously can

\footnotetext{
2 Also at Massachusetts Technological Laboratory, Inc., 330 Pleasant Street, Belmont, MA 02478, USA.

3 Also at IPST, University of Maryland, College Park, MD 20742, USA.
}

only account for $3 \mathrm{H}$ emission and is not applicable to the $4 \mathrm{H}$ or $5 \mathrm{H}$. Alternatively, the coalescence of a Langmuir wave and $2 \mathrm{H}$ EM wave was suggested for $3 \mathrm{H}$ emission, namely, $\mathrm{L}+2 \mathrm{H}$ $\rightarrow 3 \mathrm{H}$ (Zlotnik 1978). This mechanism was generalized by Cairns (1988) to explain higher harmonics including 3H, 4H, and 5H emissions. According to the scheme by Cairns (1988), higher-harmonic emission $s \mathrm{H}$ is generated as a result of merging of Langmuir and the adjacent harmonic EM wave $(s-1) \mathrm{H}$ in a cascading structure of $\mathrm{L}+2 \mathrm{H} \rightarrow 3 \mathrm{H}, \mathrm{L}+3 \mathrm{H} \rightarrow 4 \mathrm{H}$, and $\mathrm{L}+4 \mathrm{H} \rightarrow 5 \mathrm{H}$, and so forth.

An alternative mechanism for the multiple harmonic emission has been recently put forward by Yi et al. (2007). The new mechanism involves three-wave interactions among transverse EM mode and the so-called high-harmonic electrostatic (ES) nonlinear plasma waves, $\mathrm{L}+n \mathrm{~L} \rightarrow(n+1) \mathrm{H}$ (Yi et al. 2007). The modes designated by $n \mathrm{~L}$ is the $n$th harmonic ES mode generated during the beam-plasma interaction (Yoon et al. 2003). These modes are nonlinear eigenmodes in that they exist by virtue of the nonlinear response of the plasma under the influence of turbulent ES field, and they do not exist in quiescent plasmas.

As briefly outlined above, a number of theories have been put forward in order to explain the higher-harmonic emissions. The crucial issue, however, is whether the excitation of $3 \mathrm{H}$ and higher harmonics is even possible in the first place. Unless this foundational issue is first addressed, say, by means of numerical simulation experiment, the discussion of the relative efficacy of the various suggested emission mechanisms may still be subject to debate. It is the aim of this paper to carry out the detailed EM particle-in-cell (PIC) numerical simulation in two-dimensional space in order to demonstrate the high-harmonic emission, and to verify/test the various theories for high-harmonic plasma emission on the basis of the simulation results.

There is a large number of theoretical works, simulations, and a substantial body of experimental literature pertaining to the problem of beam-plasma interaction. However, numerical simulations of beam-plasma interaction process that specifically address the plasma emission have only been performed sporadically. The reason is because the plasma emission simulation requires at least two spatial dimensions and, more importantly, it requires full EM formalism that includes radiation. There are only a handful of fully two-dimensional EM simulation works 
in the literature that address the radiation emission at the fundamental and the harmonic of the plasma frequency (Pritchett \& Dawson 1983; Yin et al. 1998; Kasaba et al. 2001). In these works, the main concern was on the generation of $\mathrm{F}$ and $2 \mathrm{H}$ emission only, and the authors did not look for signatures of $3 \mathrm{H}$ or higher-harmonic components. As a consequence, none of the simulations carried out thus far report $3 \mathrm{H}$ and higherharmonic radiation. Our simulation differs from the previous efforts in that we resolve $3 \mathrm{H}$ and $4 \mathrm{H}$ radiation in addition to the $\mathrm{F} / 2 \mathrm{H}$ pair emission. We reiterate that observations of multiple harmonic emission have been reported in the literature (Reiner et al. 1992; Zlotnik et al. 1998; Cairns 1986). Consequently, our work is highly relevant to an important astrophysical problem.

\section{SIMULATION SETUP}

The simulation was carried out with a two-dimensional relativistic EM code under periodic boundary condition. The code resolves two spatial dimensions (two-dimensional) and three velocity coordinates (3V; Matsumoto \& Omura 1993). We employed $512 \times 512$ spatial grid, and the total number of time steps corresponds to $32,768\left(=2^{15}\right)$, or equivalently, $t_{\text {maximum }} \sim$ $328 \omega_{\mathrm{pe}}^{-1}$. Here, $\omega_{\mathrm{pe}}=\left(n_{e} e^{2} / \epsilon_{0} m_{e}\right)^{1 / 2}$ is the plasma frequency, with $n_{e}$ being the background electron density, $e$ being the unit electric charge, and $m_{e}$ being the electron rest mass. The grid size $\Delta x$ is taken as $\lambda_{\text {De }}$, where $\lambda_{\text {De }}=\left(\epsilon_{0} T_{e} / n_{e} e^{2}\right)^{1 / 2}$ is the Debye length, and $T_{e}$ is the electron temperature. The unit time step $\Delta t$ was taken to be equal to $0.01 \omega_{\mathrm{pe}}^{-1}$. The range of wave numbers (k) resolved in the simulation is from $0.012 \lambda_{\mathrm{De}}^{-1}$ to $\pi \lambda_{\mathrm{De}}^{-1}$. The unit in the computational system is chosen such that $\epsilon_{0}=1$.

The plasma consists of three species: the background electrons, the ions, and the electron beam. The ratio of ion-toelectron mass, $m_{i} / m_{e}$, is set close to the realistic value, namely, 1600. Thermal speed of the background electrons is $v_{e}=0.02 c$ where $c$ is the speed of light in vacuo, and $v_{e}=\left(T_{e} / m_{e}\right)^{1 / 2}$ is the thermal speed. The ion temperature is set equal to one-tenth of the background electron temperature, $T_{i}=T_{e} / 10$. The beam density $\left(n_{b}\right)$ is $2 \%$ of the background electron density, $n_{e}$; the beam drift speed $v_{d}$ is chosen to be $0.5 c$ (hence, $v_{d} / v_{e}=25$ ); and the thermal speed associated with the beam distribution is set equal to that of the background electron thermal speed, $v_{b}=v_{e}$. Here, the beam thermal speed is defined by $v_{b}=\left(T_{b} / m_{e}\right)^{1 / 2}$. In the previous simulation of a relatively lower beam speed, $0.2 c$ (Kasaba et al. 2001), only $F$ and $2 \mathrm{H}$ are observed. By increasing the beam velocity, the present case allows for the excitation of higher-harmonic modes. We find that at the minimum, $v_{d} / c=0.5$ is necessary in order for the higher-harmonic excitation to take place. It is generally accepted that typical beam speed associated with type III radio bursts ranges from $0.2 c$ to $0.5 c$. Thus, $v_{d} / c=0.5 c$ is on the high end of this range. If we take the beam velocity to be higher, then we not only enter unrealistic parameter regime but also from a theoretical standpoint we encroach upon the territory of strong turbulence where the physical interpretation of decay and merging of waves may not be too meaningful. For this reason, we confine ourselves to $v_{d} / c=0.5$ throughout the present study.

Velocity distribution functions for the background electrons and ions are assumed to be isotropic Maxwellian, while the beam electron distribution is modeled by the drifting Maxwellian. The plasma is weakly magnetized such that the cyclotron frequency is $1 \%$ of the plasma frequency, $\Omega_{\mathrm{ce}}=0.01 \omega_{\mathrm{pe}}$. In a typical solar wind, the ratio $\Omega_{\mathrm{ce}} / \omega_{\mathrm{pe}}$ is on the order of $\sim 10^{-2}$ (Parks 2003). Hence, this choice represents a fairly typical situation. The present simulation employs 21 million background electrons, 2.1 million beam particles, and 21 million ions. We have assigned one-fifth of the electric charge and mass for the beam particles, i.e., their charge and mass are $(1 / 5) e$ and $(1 / 5) m_{e}$ of those of the background electrons. That is, each beam electron is represented by five beam particles. Splitting the beam electrons to particles with smaller charge and mass while preserving $e / m_{e}$ is often adopted in typical PIC simulations to enhance the statistical accuracy (Pritchett \& Dawson 1983). Initially, the background electrons, the beam particles, and the ions are uniformly distributed.

\section{RESULTS}

According to the present simulation, the beam-plasma interaction takes place in roughly three distinct stages: the initial, interactive, and nonlinear phases. The panels on the left in Figure 1 show the time evolution of the particle energy associated with the background electrons $\left(E_{\text {b.g. }}\right)$, the particle (kinetic plus thermal) energy of the beam particles $\left(E_{\text {beam }}\right)$, and the electric field energy $\left(E_{\mathrm{E}}\right)$, plotted versus the normalized time $\omega_{\mathrm{pe}} t$. The right-hand panels display the $x-v_{x}$ phase space plot at the times $\omega_{\mathrm{pe}} t=20.48,61.44$, and 245.76. These times are indicated by the arrows on the upper left-hand panel. Here, the normalized spatial coordinate $X$ represents the $x$-axis in the simulation box, and the velocity is normalized by the speed of light in vacuo $c$.

In the initial phase, energy exchange between the background and beam electrons as well as the electric field is not apparent (see the left-hand panels in the temporal range $0<\omega_{\text {pe }} t<35$, albeit the exact time designation is somewhat arbitrary), as the two electron species occupy nonoverlapping regions in the phase space (top-right panel). In the interactive stage (35 $<\omega_{\mathrm{pe}} t<100$, again this time designation is somewhat arbitrary), the beam-plasma interaction is shown to proceed. First, in this stage, it can be seen that roughly $40 \%$ of the beam energy is transferred to the background electron energy and to the $E$ field energy. Second, as shown in the phase-space plot at $61.44 \omega_{\mathrm{pe}} t$, the beam electrons have spread out in velocity space, and the characteristic phase-space vortices or whirls that typify the beam-plasma interaction can be seen to form. In the nonlinear phase, beyond $\omega_{\mathrm{pe}} t=100$, it can be seen that phase-space vortices have completely randomized. As shown in the bottom-right plot at $245.76 \omega_{\mathrm{pe}} t$, the beam and background electrons have intermingled in the phase space so that it is no longer meaningful to distinguish the two species. The plasma can be said to have entered the genuinely turbulent state.

We next examine the generation of multiple EM radiation during the beam-plasma interaction process. Figures 2(a) and (b) respectively show simulated $\omega-k_{x}$ diagram for ES and EM modes, by projecting wave intensities along the wavenumber component parallel to the beam propagation direction $\left(k_{x}\right.$-axis). ES and EM modes were obtained by fast Fourier transformation (FFT) of $E_{x}$ and $B_{z}$ field over the period ranging from $t \omega_{\mathrm{pe}}=$ 246 to $\sim 328$. Frequency and wavenumber are respectively normalized according to $\omega / \omega_{\text {pe }}$ and $c k_{x} / \omega_{\text {pe }}$. Henceforth, the spectral intensities in this paper are all normalized with respect to the electron thermal energy, $n_{e} T_{e}$, and the intensity level is indicated by the color bar.

Distinctly visible in Figure 2(a) are enhanced wave intensities in $\omega-k_{x}$ space that correspond to the fundamental Langmuir 

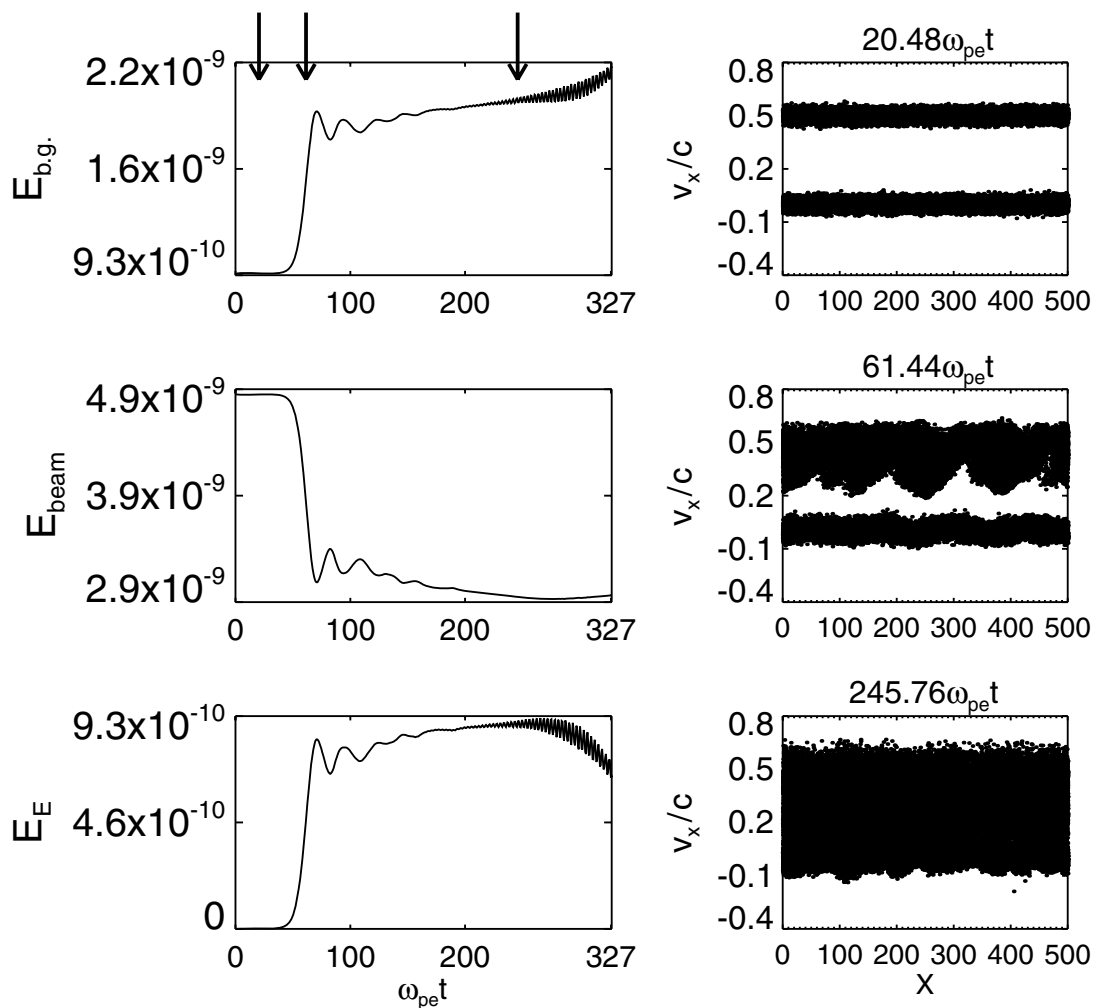

Figure 1. Time evolution of the particle (background and beam) energy and $E$ field energy (left-hand panels), and snapshots of the $x-v_{x}$ phase space at 20.48, 61.44, and $245.76 \omega_{\mathrm{pe}} t$.

wave and its harmonics. The Langmuir wave dispersion relation,

$$
\omega / \omega_{\mathrm{pe}}=1+3 k^{2} \lambda_{\mathrm{De}}^{2} / 2
$$

is superposed in Figure 2(a) in red. The enhanced intensity at each harmonic of $\omega_{\mathrm{pe}}$ ranging from the fundamental plasma frequency up to $4 \omega_{\text {pe }}$ can be observed. The parallel component of the phase speed associated with these modes is correlated with the beam speed,

$$
\omega / k_{x}=0.5 c,
$$

which is also indicated in red. Yoon et al. (2003) analyzed such ES harmonic modes in detail, and they showed that these are nonlinear eigenmodes that exist in turbulent plasmas. These high-harmonic ES modes cannot escape to free space in uniform plasmas, and thus they do not directly contribute to the radiation at multiple-harmonic plasma frequency. Note that the (fundamental) Langmuir and multiple-harmonic ES modes possess broad spectra along the $k_{x}$-axis, indicating that these modes are incoherent waves. These modes turn out to be propagating predominantly along the $k_{x}$-axis, as we shall confirm this in Figure 3.

Backscattered (fundamental) Langmuir modes that exist in negative $k_{x}$ space are the result of decay and induced scattering processes involving either the ion-acoustic mode or the thermal ions. The generation of a low-frequency spectrum can also be discerned. We interpret this mode as corresponding to the ionsound wave. The presence of the ion-sound mode is often cited as evidence for the nonlinear decay process, although the decay may proceed even when the level of ion-sound waves may not be immediately apparent (Ziebell et al. 2001). In the present simulation, the excitation of the ion-sound wave indicates that the backscattered Langmuir wave is produced by the decay process. We were not able to verify whether the competing
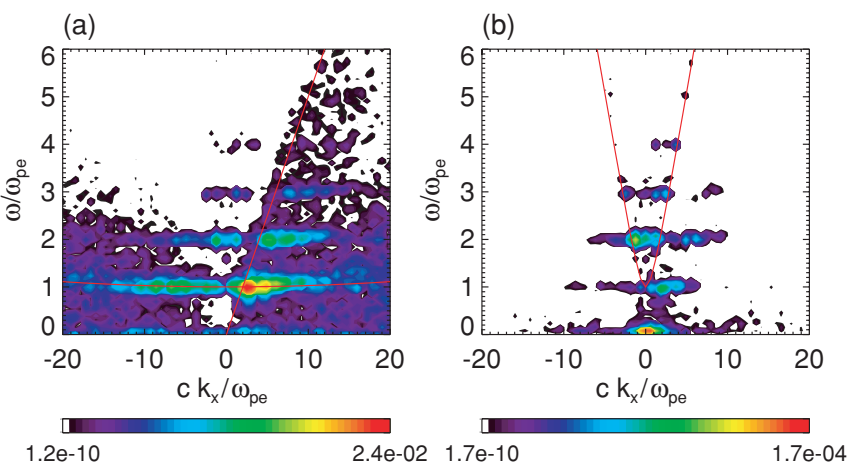

$2.4 \mathrm{e}-02 \quad 1.7 \mathrm{e}-10$

$1.7 e-04$

Figure 2. Numerical $\omega-k_{x}$ "dispersion relation" generated from $E_{x}(a)$ and $B_{z}(b)$ field, plotted in frequency vs. wavenumber along the beam-propagation direction, at $t \omega_{\text {pe }}=246-328$.

(A color version of this figure is available in the online journal.)

process of induced scattering off thermal ions is operative or not since it is difficult to quantify the ion-scattering process within the present context of the particle simulation.

In addition to the nonlinear ES harmonic modes in the regime

$$
\omega / k_{x}<0.5 c,
$$

also note that $2 \mathrm{H}, 3 \mathrm{H}$, and $4 \mathrm{H} E_{x}$ modes exist in the longwavelength regime satisfying the condition

$$
\omega / k_{x}>0.5 c .
$$

However, these modes are not nonlinear ES harmonics propagating parallel to the $x$-axis, but it turns out that they are obliquely propagating EM harmonic modes. Figure 2(a) is simply the result of the projection effects. That the EM harmonic waves have oblique angles of propagation will be confirmed later. 

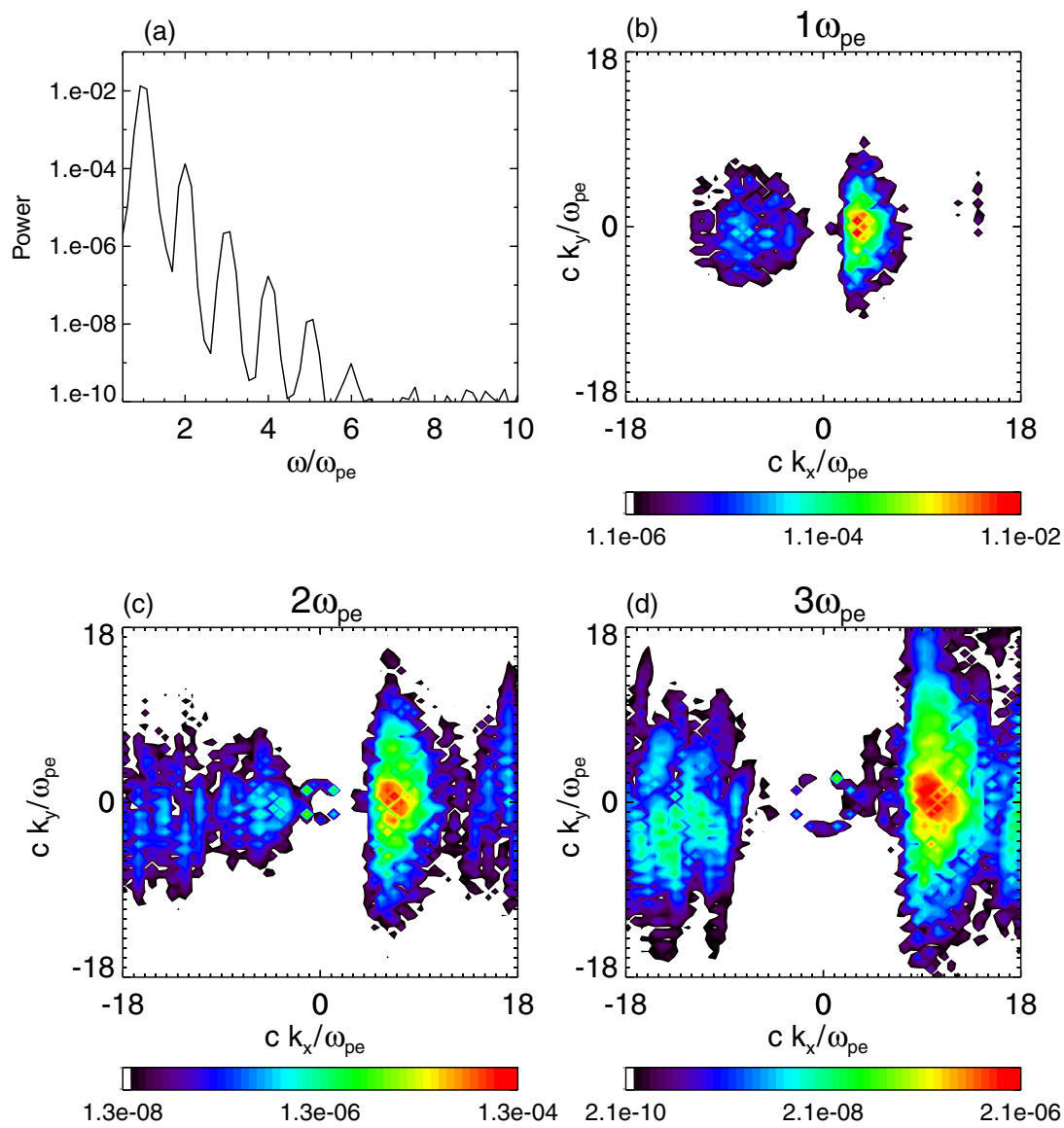

Figure 3. (a) Peak power associated with $E_{x}(\omega, k)$ spectrum plotted against the normalized frequency, and plots of the $E_{x}$ field at frequency in the vicinity of $(b) \omega_{\text {pe }}$, (c) $2 \omega_{\text {pe }}$, and (d) $3 \omega_{\text {pe }}$ vs. $k_{x}$ and $k_{y}$.

(A color version of this figure is available in the online journal.)

Turning to Figure 2(b), observe the discrete enhancements of EM wave intensities at multiples of plasma frequency up to $4 \mathrm{H}$ or even $5 \mathrm{H}$. It turns out that these EM harmonic modes possess phase speeds approximately equal to the speed of light in vacuo, even though they appear to have phase speeds exceeding $c$. This is again the result of projecting the two-dimensional results onto the $k_{x}$-axis. We have superposed the conventional EM dispersion relation,

$$
\omega^{2}=\omega_{\mathrm{pe}}^{2}+k^{2} c^{2},
$$

in the red curve. Portions of EM harmonic modes having parallel phase speeds in excess of the speed of light,

$$
\omega / k_{x}>c,
$$

are actually customary transverse EM waves satisfying the above EM dispersion relation. These modes have oblique directions of propagation, as will be discussed later.

Portions of EM harmonics satisfying

$$
\omega / k_{x}<c,
$$

on the other hand, do not satisfy the above EM dispersion relation. Consequently, these modes cannot be discussed on the basis of the customary textbook theory. Such harmonic EM waves with short wavelengths and with frequency $\sim n \omega_{\text {pe }}$ have been analyzed by Yoon (1995) and Yoon et al. (2005). These modes are EM counterparts of the ES nonlinear eigenmodes discussed earlier. Consequently, Yoon (1995) and Yoon et al. (2005) have provided essential explanations for the existence of these modes. However, the analyses by Yoon (1995) and Yoon et al. (2005) are primarily concerned with the superluminal regime of the nonlinear EM harmonics, $\omega / k>c$, so strictly speaking the EM harmonics in the subluminal regime, $\omega / k<c$, have not been studied in detail.

Figure 3 consists of four panels. Figure 3(a) displays the frequency spectrum of the ES mode. Figure 3(a) was generated by taking maximum intensity along the wavenumber $k_{x}$, for a given frequency $\omega$. We have then varied $\omega$ in order to produce the power spectrum in frequency. This figure is thus a further projection of Figure 3(a) onto the vertical frequency axis. It can be seen from Figure 3(a) that ES multiple harmonic modes, visible up to the sixth harmonic $(6 \mathrm{H})$, can be characterized by a steep power-law spectrum in frequency space. The intensity of ES harmonics decreases by 2 orders in magnitude for every increase in the harmonic mode number.

Shown in Figures 3(b)-(d) are plots of electric field $E_{x}$ versus $k_{x}$ and $k_{y}$, at fixed frequencies corresponding to $\omega_{\mathrm{pe}}, 2 \omega_{\mathrm{pe}}$, and $3 \omega_{\text {pe }}$, respectively. Figure 3(b) thus represents the fundamental Langmuir mode intensity plotted in two-dimensional wavenumber space, Figure 3(c) corresponds to the $2 \mathrm{H}$ ES mode, and Figure 3(d) to the $3 \mathrm{H}$ ES mode. The wave spectra were generated by collecting simulation data during the time period from $\omega_{\mathrm{pe}} t=287$ to $\omega_{\mathrm{pe}} t=328$. Although we have loosely interpreted these modes as ES modes they actually possess mixed EM/ES polarization, particularly for small $k$ values. These 
(a)

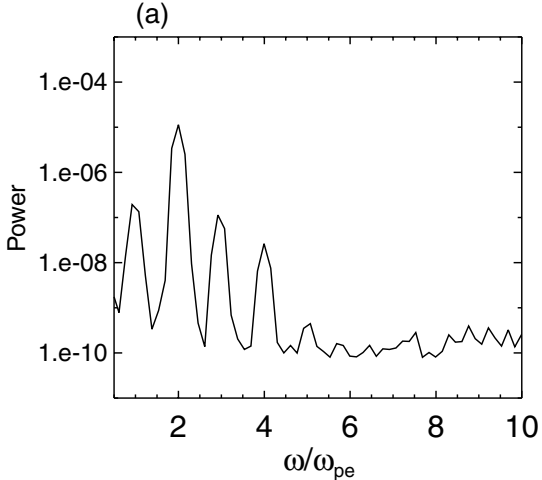

(c)

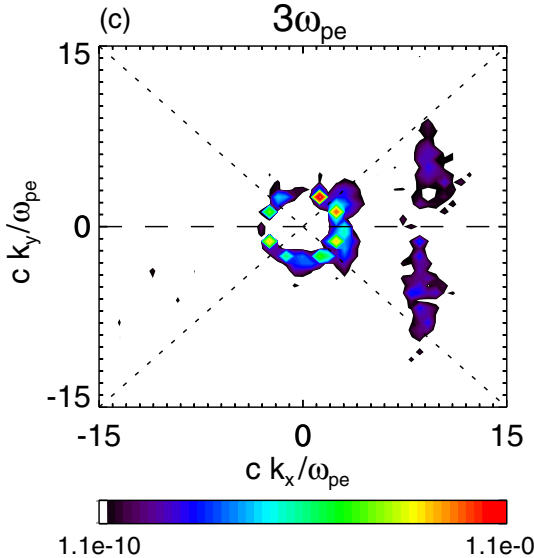

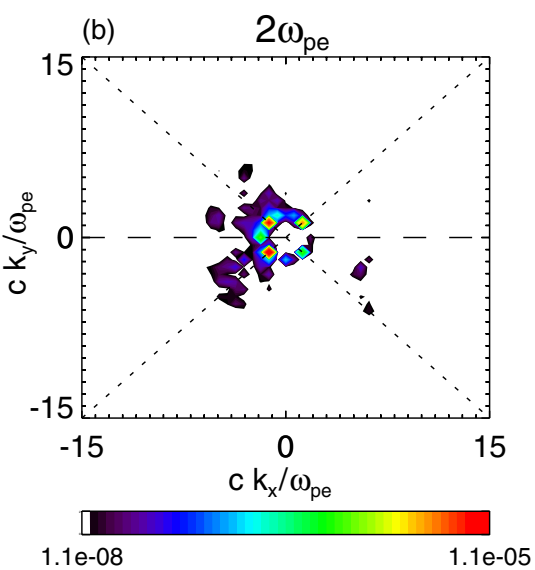

(d)

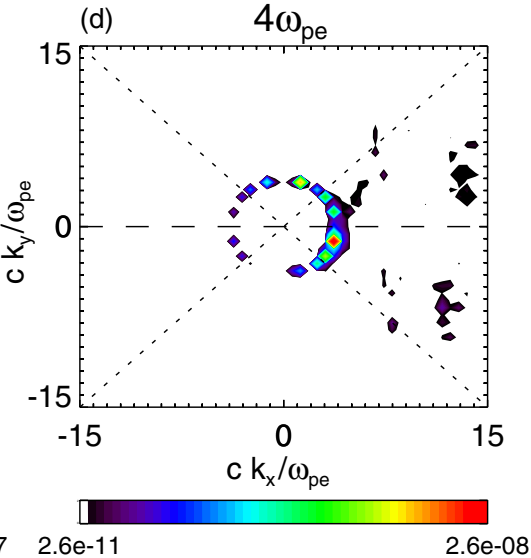

Figure 4. (a) Peak power associated with $B_{z}(\omega, k)$ spectrum plotted against the normalized frequency, and plots of the $B_{z}$ field at frequency in the vicinity of $(b) 2 \omega_{\text {pe }}$, (c) $3 \omega_{\mathrm{pe}}$, and (d) $4 \omega_{\text {pe }}$ vs. $k_{x}$ and $k_{y}$.

(A color version of this figure is available in the online journal.)

modes are purely ES only along a line defined by $k_{y}=0$ in the two-dimensional wavenumber space.

Short-wavelength portions of the displayed $E_{x}$ field have peak wave intensities along $k_{x}$-axis. This confirms our earlier assertion that fundamental Langmuir and ES nonlinear eigenmodes are propagating parallel to the beam. However, the $E_{x}$ field near the origin in two-dimensional wavenumber space that contain large EM components has peak intensity along small circular ring structures with increasing radius moving from Figures 3(b) to (d) (for Figure 3(b) the ring is virtually a dot). These longwavelength EM harmonic modes, therefore, do not have parallel propagation direction, but are in fact oblique modes. We shall discuss the properties of EM harmonic modes in more detail later when we discuss Figure 4.

The primary Langmuir wave spectrum shown in Figure 3(b) has an arc shape, with the wave energy being concentrated in the range, $1.2 \leqslant k_{x} c / \omega_{\text {pe }} \leqslant 8$ and $\left|k_{y} c / \omega_{\text {pe }}\right| \leqslant 8$. The width of the spectrum along the $k_{y}$-axis is much broader than along $k_{x}$. The backscattered Langmuir mode (negative $k_{x}$ ) is seen to occupy a broad region although the intensity level is much lower. The specific range of the backscattered mode roughly corresponds to $-12 \leqslant k_{x} c / \omega_{\text {pe }} \leqslant-1.2$ and $\left|k_{y} c / \omega_{\text {pe }}\right|<6$. The backscattered mode has a Gaussian profile rather than an arc shape spectral distribution.

The wave intensity associated with the $2 \mathrm{H}$ ES mode is comparable to that of the backscattered Langmuir wave. Forwardpropagating components of both the second and third ES harmonic modes possess arc-shaped spectra in two-dimensional wavenumber space. Figure 3(c) shows that the range of the ES $2 \mathrm{H}$ mode corresponds to $4.8 \leqslant k_{x} c / \omega_{\mathrm{pe}} \leqslant 12$ and $\left|k_{y} c / \omega_{\mathrm{pe}}\right|<$ 13. In the long-wavelength regime (small circular structure near the center), a quadrupole pattern can be discerned. As noted earlier, this structure corresponds to the EM $2 \mathrm{H}$ mode. The $3 \mathrm{H}$ ES mode lies in the range $7 \leqslant k_{x} c / \omega_{\text {pe }} \leqslant 16$ and $\mid k_{y} \lambda$ De $\mid<18$ (Figure 3(d)). As with Figure 3(c), the EM component of the 3H $E_{x}$ field is shown as a ring structure with slightly larger radius.

The $2 \mathrm{H}$ and $3 \mathrm{H}$ ES modes shown in Figures 3(c) and (d), have backscattered components, although when projected onto the $k_{x}$-axis, these modes are too weak to show up clearly in Figure 2(a). The theories by Yoon (1995) and Yoon et al. (2005) cannot yet be extended to discuss such backscattered harmonic modes. Thus, no satisfactory theory exists to account for the apparent backscattering of these modes.

In Figure 4, we represent the EM mode by plotting the $B_{z}$ field in various formats. First, the frequency spectrum of the EM mode is shown in Figure 4(a). Multiple harmonic EM emission up to fifth can be identified. The spectral peak associated with the fifth harmonic can be distinguished from the background noise even though it is rather low. The highest wave intensity is associated with the $2 \mathrm{H} \mathrm{EM}$ emission, indicating that the plasma emission should be dominated by the $2 \mathrm{H}$ component-at least for the present set of input parameters. The next significant emission occurs at the $F$ component.

Figures 4(b)-(d) display detailed structures associated with each harmonic EM mode in two-dimensional wavenumber 
space. We have chosen not to display the fundamental emission spectrum, since the emission pattern appears virtually as a dot over the range of two-dimensional $k$ vector space. Figure 4(b) shows the radiation pattern associated with the $2 \mathrm{H} \mathrm{EM}$ mode. The enhanced emission is seen to take place along the circumference of a circular ring spectrum. A careful examination shows that the angles corresponding to peak emission correspond to

$$
\theta \sim 45^{\circ} \text { and } \theta \sim 135^{\circ}
$$

in the upper-half two-dimensional $k$ space, and a similar radiation pattern in the lower two-dimensional $k$ space. Lower quadrants in Figure 4(b) are symmetric when compared with the upper quadrants. Here, the radiation angle $\theta$ is defined with respect to the direction of the beam propagation.

Figures $4(\mathrm{c})$ and (d) correspond to $3 \mathrm{H}$ and $4 \mathrm{H}$ radiation emission. These higher-harmonic EM emissions have never been investigated in previous EM beam-plasma interaction simulations. Figure 4(c) shows the $3 \mathrm{H}$ EM radiation. Note that the angle of peak radiation occurs along

$$
\theta \sim 27^{\circ}, \sim 63^{\circ}, \text { and } \sim 153^{\circ} .
$$

We shall discuss the physics behind such a radiation pattern later. The $4 \mathrm{H}$ EM radiation is shown in Figure 4(d). The peak emission is observed to take place at angles

$$
\theta \sim 20^{\circ}, \sim 40^{\circ}, \text { and } \sim 70^{\circ} .
$$

With the exception of $\sim 70^{\circ}$, the radiation angle is directed largely parallel to the beam-propagation direction. Note that backward radiation is practically absent at the $4 \mathrm{H}$ plasma frequency.

From the above results, radiation pattern at each harmonic of the plasma frequency is quite distinct from each other. These results have an important practical implication in that the power spectrum of the observed multiple harmonics will be different at the different measuring positions. According to some reported observations (Reiner et al. 1992; Zlotnik et al. 1998), the $3 \mathrm{H}$ component is weaker than the $2 \mathrm{H}$ radiation. On the other hand, other observations report more intense $3 \mathrm{H}$ emission than the $2 \mathrm{H}$ component (Takakura \& Yousef 1974). This conflicting relative difference in the radiation intensity has been the source of some controversy (Zlotnik 1978). According to our findings, however, the relative intensity of the harmonic radiation might be highly dependent on the measuring angle.

\section{INTERPRETATION}

We now use the difference in the radiation patterns at multiple harmonics determined from the present simulation to test and verify the available high-harmonic radiation theories. The various theories proposed thus far make their own predictions regarding the characteristic radiation patterns. Thus, the present simulation provides a valuable testing ground for the available theories for higher-harmonic emission.

The $2 \mathrm{H}$ radiation theory is well known (Willes et al. 1996; Yoon 2006). The amplification of radiation is dictated by the wave kinetic equation

$$
\begin{aligned}
\frac{\partial I_{T}(\mathbf{k})}{\partial t}= & \frac{\pi e^{2}}{m_{e}^{2} \omega_{\mathrm{pe}}^{2}} \int d \mathbf{k}^{\prime} \frac{\left(\mathbf{k} \times \mathbf{k}^{\prime}\right)^{2}\left(\mathbf{k} \cdot \mathbf{k}^{\prime}\right)^{4}}{k^{2} k^{\prime 4}} \\
& \times I_{L}\left(\mathbf{k}^{\prime}\right) I_{L}\left(\mathbf{k}-\mathbf{k}^{\prime}\right) \delta\left(\omega_{\mathbf{k}}^{T}-\omega_{\mathbf{k}^{\prime}}^{L}-\omega_{\mathbf{k}-\mathbf{k}^{\prime}}^{L}\right)
\end{aligned}
$$

where $I_{L}(\mathbf{k})$ and $I_{T}(\mathbf{k})$ are the spectral intensities associated with the Langmuir and EM modes. For isotropic Langmuir wave intensities, the radiation angular pattern for the $2 \mathrm{H}$ EM mode is determined largely by the transition probability, $\left(\hat{\mathbf{k}} \times \hat{\mathbf{k}}^{\prime}\right)^{2}\left(\hat{\mathbf{k}} \cdot \hat{\mathbf{k}}^{\prime}\right)^{4} \sim \sin ^{2} \theta \cos ^{4} \theta$. (Here, we make the simplifying assumption of isotropic Langmuir wave intensity for the sake of simplicity, although Figure 3(b) shows that the actual Langmuir wave intensity is highly angle dependent.) This shows that the radiation intensity should be approximately the same for $45^{\circ}$ and $135^{\circ}$. Our simulation shows, however, that the backward radiation at $135^{\circ}$ is slightly more intense than the forward radiation at $45^{\circ}$. This can be explained by the fact that we have made the simplifying assumption of the isotropic Langmuir wave spectrum, when in reality, the Langmuir wave spectrum is anisotropic. In their numerical study, Willes et al. (1996) assumed asymmetric forward versus backward Langmuir wave intensities, which is in agreement with our simulation result. However, we should note that the Langmuir waves are still evolving at the end of our simulation and have not reached the truly stationary stage. Thus, the assumption of isotropic spectrum may become valid over longer time period than our simulation time span. In short, the customary theory of $2 \mathrm{H}$ emission is in good agreement with our simulation.

We now test the two theories proposed for the higherharmonic radiation. As noted, one theory involves the generation of higher-harmonic EM modes by merging of Langmuir and EM waves, $\mathrm{L}+2 \mathrm{H} \rightarrow 3 \mathrm{H}$ and $\mathrm{L}+3 \mathrm{H} \rightarrow 4 \mathrm{H}$ (Zlotnik 1978; Cairns 1988). The other theory involves the merging process $\mathrm{L}+n \mathrm{~L} \rightarrow$ $\mathrm{T}$ (Yi et al. 2007). To compare the simulation results with the theory involving the coalescence of Langmuir and EM waves, we note that the radiation pattern predicted by this theory is dictated by the following equation (Zlotnik 1978; Cairns 1988):

$$
\begin{aligned}
\frac{\partial I_{T}(\mathbf{k})}{\partial t}= & -\frac{\pi e^{2} \omega_{\mathrm{pe}}^{2}}{4 m_{e}^{2}} \int d \mathbf{k}^{\prime} \frac{\left|\mathbf{k}-\mathbf{k}^{\prime}\right|^{2}}{\omega_{\mathbf{k}}^{T} \omega_{\mathbf{k}^{\prime}}^{T}}\left(1+\frac{\left(\mathbf{k} \cdot \mathbf{k}^{\prime}\right)^{2}}{k^{2} k^{\prime 2}}\right) \\
& \times I_{L}\left(\mathbf{k}-\mathbf{k}^{\prime}\right) I_{T}\left(\mathbf{k}^{\prime}\right) \delta\left(\omega_{\mathbf{k}}^{T}-\omega_{\mathbf{k}^{\prime}}^{T}-\omega_{\mathbf{k}-\mathbf{k}^{\prime}}^{L}\right) .
\end{aligned}
$$

The $3 \mathrm{H}$ radiation at the frequency $3 \omega_{\mathrm{pe}}$ is described by the interaction between Langmuir and $2 \mathrm{H}\left(2 \omega_{\mathrm{pe}}\right) \mathrm{EM}$ waves. In the above equation, $3 \mathrm{H}$ intensity, $I_{T}(\mathbf{k})$, is obtained by choosing the value of $\mathbf{k}$ vector corresponding to $\mathrm{EM}$ wave dispersion at $\omega=3 \omega_{\text {pe }}$. This leads to $|\mathbf{k}|=2 \sqrt{2} \omega_{\text {pe }} / c$ with frequency at $3 \omega_{\text {pe }}$. In the same vein, the $\left|\mathbf{k}^{\prime}\right|$ vector for the $2 \mathrm{H}$ intensity, $I_{T}\left(\mathbf{k}^{\prime}\right)$, is fixed at $k^{\prime}=\sqrt{3} \omega_{\text {pe }} / c$ at $\omega=2 \omega_{\text {pe }}$. Therefore, the value of $\left|\mathbf{k}-\mathbf{k}^{\prime}\right|$ lies in the range

$$
\frac{\omega_{\mathrm{pe}}}{c}(2 \sqrt{2}-\sqrt{3})<\left|\mathbf{k}-\mathbf{k}^{\prime}\right|<\frac{\omega_{\mathrm{pe}}}{c}(2 \sqrt{2}+\sqrt{3}) .
$$

The term within the large parentheses in the above equation ranges from 1 to 2 depending on the angular factor $(\mathbf{k}$. $\left.\mathbf{k}^{\prime}\right)^{2} / k^{2} k^{\prime 2}=\cos ^{2}\left(\theta-\theta^{\prime}\right)$. Here, we have designated $\theta$ and $\theta^{\prime}$ to denote propagation angles for $\mathbf{k}$ and $\mathbf{k}^{\prime}$ vectors, respectively. The argument of the Langmuir wave intensity, $\left|\mathbf{k}-\mathbf{k}^{\prime}\right|$, must be determined according to the delta function resonance condition. The Langmuir wave frequency is practically $\omega \sim \omega_{\mathrm{pe}}$ over the entire range so that the delta function is trivially satisfied. Thus, the remaining factor that plays the key role in determining the radiation angle is the product of the two intensities, $I_{L}(\mathbf{k}-$ $\left.\mathbf{k}^{\prime}\right) I_{T}\left(\mathbf{k}^{\prime}\right)$.

The wave vector associated with the $3 \mathrm{H}$ mode $\mathbf{k}$ must be determined when the product of the two intensities, $I_{L}\left(\mathbf{k}-\mathbf{k}^{\prime}\right) I_{T}\left(\mathbf{k}^{\prime}\right)$, 
is maximum. The maximum peak associated with the Langmuir wave occurs at $\theta \sim 0^{\circ}$ and $180^{\circ}$. The maximum peak of the second EM wave, on the other hand, takes place when $\theta \sim 45^{\circ}$ and $135^{\circ}$. If we denote the cosine of the angle associated with the vector $\mathbf{k}-\mathbf{k}^{\prime}$ as $\cos \gamma$, and if we represent the vectors $\mathbf{k}$ and $\mathbf{k}^{\prime}$ as $(k, \theta)$ and $\left(k^{\prime}, \theta^{\prime}\right)$, respectively, then we have $\cos \gamma=\left(k \cos \theta-k^{\prime} \cos \theta^{\prime}\right) /\left|\mathbf{k}-\mathbf{k}^{\prime}\right|$. The magnitudes $k$ and $k^{\prime}$ can be estimated to be $2 \sqrt{2} \omega_{\mathrm{pe}} / c$ and $\sqrt{3} \omega_{\mathrm{pe}} / c$, respectively, and $\left|\mathbf{k}-\mathbf{k}^{\prime}\right|$ can be determined to lie between $(2 \sqrt{2}-\sqrt{3}) \omega_{\text {pe }} / c$ and $(2 \sqrt{2}+\sqrt{3}) \omega_{\mathrm{pe}} / c$. From this, we determine that the cosine angle of the vector $\mathbf{k}-\mathbf{k}^{\prime}$ must be

$$
\frac{2 \sqrt{2} \cos \theta-\sqrt{3} \cos \theta^{\prime}}{2 \sqrt{2}+\sqrt{3}} \leqslant \cos \gamma \leqslant \frac{2 \sqrt{2} \cos \theta-\sqrt{3} \cos \theta^{\prime}}{2 \sqrt{2}-\sqrt{3}} .
$$

Obviously, the absolute values of the lower and upper limits in the above equation cannot exceed 1 . This limitation is implicit in the above relation.

Let us first consider the case when the wavenumber for the Langmuir wave, $\mathbf{k}-\mathbf{k}^{\prime}$, has $0^{\circ}$ propagation angle, or $\cos \gamma \sim 1$. Taking into account that the peak angles for $\mathbf{k}^{\prime}$ vector associated with the second EM wave have two values, namely, $\theta^{\prime} \sim 45^{\circ}$ and $\theta^{\prime} \sim 135^{\circ}$, we immediately see that the angle $\theta$ associated with the $\mathbf{k}$ vector ( $3 \mathrm{H} \mathrm{EM}$ mode) having a value $\sim 27^{\circ}$ easily satisfies the relation (1) with $\cos \gamma \sim 1$ for both $\theta^{\prime}=45^{\circ}$ and $\theta^{\prime}=135^{\circ}$. A similar argument shows that the case of $\gamma \sim 180^{\circ}$ or $\cos \gamma \sim-1$ works equally well for both $\theta^{\prime}=45^{\circ}$ and $\theta^{\prime}=135^{\circ}$ as long as $\theta \sim 153^{\circ}$.

However, in the case of $\theta \sim 63^{\circ}$, relation (1) can be satisfied only if $\theta^{\prime}=135^{\circ}$ and $\gamma \sim 0^{\circ}$. It fails for $\gamma \sim 180^{\circ}$, however, regardless of whether $\theta^{\prime} \sim 45^{\circ}$ or $135^{\circ}$.

In short, the mechanism proposed by Zlotnik (1978) and generalized by Cairns (1988) can qualitatively explain the radiation emission at $3 \mathrm{H}$ when the radiation angle corresponds to $\theta \sim 27^{\circ}, 153^{\circ}$. However, for $\theta \sim 63^{\circ}$, the validity of their theory is somewhat questionable.

The $4 \mathrm{H}$ radiation according to Zlotnik-Cairns mechanism works when the Langmuir and $3 \mathrm{H}$ EM mode interacts via three-wave coalescence. Elementary calculation shows that $k^{\prime} \sim 2 \sqrt{2} \omega_{\text {pe }} / c$ with $\omega^{\prime} \sim 3 \omega_{\text {pe }}$ and $k \sim \sqrt{15} \omega_{\text {pe }} / c$ with $\omega \sim 4 \omega_{\text {pe }}$. Consequently, the value of $\left|\mathbf{k}-\mathbf{k}^{\prime}\right|$ lies in the range

$$
\frac{\omega_{\mathrm{pe}}}{c}(\sqrt{15}-2 \sqrt{2})<\left|\mathbf{k}-\mathbf{k}^{\prime}\right|<\frac{\omega_{\mathrm{pe}}}{c}(\sqrt{15}+2 \sqrt{2}),
$$

which is consistent with the simulation result. The cosine angle associated with the vector $\mathbf{k}-\mathbf{k}^{\prime}$ must therefore lie in the range specified by

$$
\frac{\sqrt{15} \cos \theta-2 \sqrt{2} \cos \theta^{\prime}}{\sqrt{15}+2 \sqrt{2}} \leqslant \cos \gamma \leqslant \frac{\sqrt{15} \cos \theta-2 \sqrt{2} \cos \theta^{\prime}}{\sqrt{15}-2 \sqrt{2}} .
$$

Let us first consider the $4 \mathrm{H} \mathrm{EM}$ emission at angle $20^{\circ}$. Recall that the choices of $\gamma$ are limited to $\gamma \sim 0^{\circ}$ and $\gamma \sim 180^{\circ}$, while $\theta^{\prime}$ is limited to $\theta^{\prime} \sim 27^{\circ}, \sim 63^{\circ}$, and $153^{\circ}$ only. For this case, combinations of $\left(\gamma, \theta^{\prime}\right) \sim\left(0^{\circ}, 27^{\circ}\right)$ and $\left(\gamma, \theta^{\prime}\right) \sim\left(0^{\circ}, 63^{\circ}\right)$ satisfy relation (2), but other combinations do not work.

In the case of $4 \mathrm{H} \mathrm{EM}$ emission at $\theta \sim 40^{\circ}$, the only combination that satisfies relation (2) is when $\left(\gamma, \theta^{\prime}\right) \sim\left(0^{\circ}, 63^{\circ}\right)$. No other combined values of $\gamma$ and $\theta^{\prime}$ can satisfy Equation (2). Finally, in the case of emission at $\theta \sim 70^{\circ}$, there are two possible combinations of angles that satisfy Equation (2). These are $\left(\gamma, \theta^{\prime}\right) \sim\left(180^{\circ}, 27^{\circ}\right)$ and $\left(\gamma, \theta^{\prime}\right) \sim\left(0^{\circ}, 153^{\circ}\right)$.
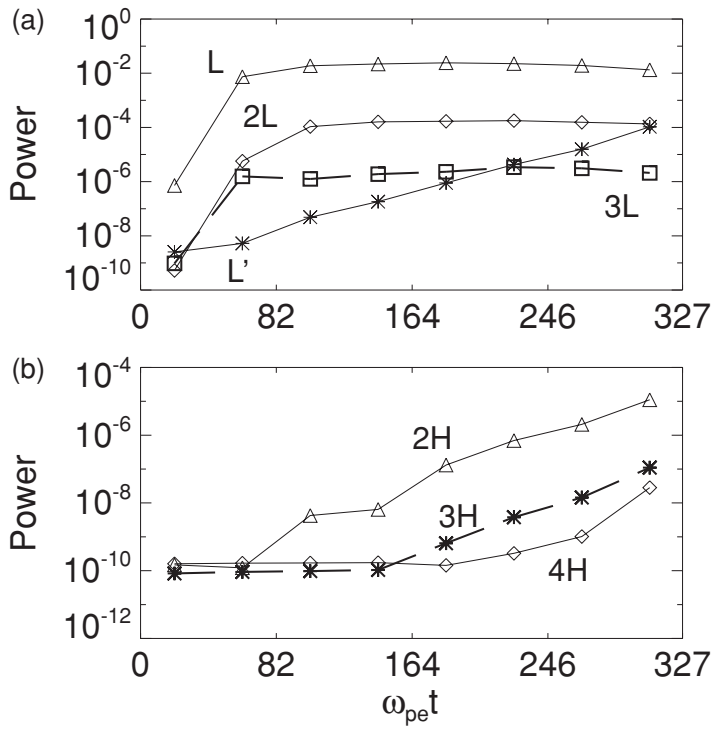

Figure 5. Time evolution of (a) electrostatic harmonics L, 2L, 3L, and backscattered Langmuir wave $\mathrm{L}^{\prime}$, and $(b)$ electromagnetic harmonics $2 \mathrm{H}, 3 \mathrm{H}$, and $4 \mathrm{H}$. Yi et al.'s model pertains to the generation of ES harmonics $(a)$, while Zlotnik-Cairns theory is applicable for EM harmonics $(b)$.

The foregoing discussion again shows that Zlotnik-Cairns mechanism qualitatively explains $4 \mathrm{H}$ emission. In the alternative theory proposed by Yi et al. (2007), the multiple harmonic EM emission takes place as a result of three-wave interaction that involves ES nonlinear harmonics, $\mathrm{L}+n \mathrm{~L} \rightarrow(n+1) \mathrm{H}$. The growth of the harmonic EM wave is dictated by

$$
\begin{aligned}
\frac{\partial I_{T}(\mathbf{k})}{\partial t}= & \frac{\pi e^{2}}{16 m_{e}^{2} \omega_{\mathrm{pe}}^{2}} \int d \mathbf{k}^{\prime} \frac{k^{\prime 2}}{k^{2}}\left(\mathbf{k} \times \mathbf{k}^{\prime}\right)^{2} \\
& \times I_{n L}\left(\mathbf{k}^{\prime}\right) I_{L}\left(\mathbf{k}-\mathbf{k}^{\prime}\right) \delta\left(\omega_{\mathbf{k}}^{T}-\omega_{\mathbf{k}^{\prime}}^{n L}-\omega_{\mathbf{k}-\mathbf{k}^{\prime}}^{L}\right),
\end{aligned}
$$

where $I_{n L}\left(\mathbf{k}^{\prime}\right)$ stands for the wave intensity associated with the ES $n$th harmonic mode. In this theory, the overall angular dependence associated with the radiation at the $n$th harmonic is simply dictated by the factor $\left(\mathbf{k} \times \mathbf{k}^{\prime}\right)^{2}$ multiplied by the angular distributions associated with the two intensities, $I_{n L}\left(\mathbf{k}^{\prime}\right)$ and $I_{L}\left(\mathbf{k}-\mathbf{k}^{\prime}\right)$. Both intensities have peak values when the propagation angles associated with $\mathbf{k}^{\prime}$ and $\mathbf{k}-\mathbf{k}^{\prime}$ are aligned with the beam-propagation direction. Some careful analyses reveal that the radiation pattern predicted by Yi et al. mechanism is not consistent with the present simulation results.

Up to this point, we have compared the characteristics of the angle dependence of the multiple harmonic radiation to available theories. Next, we wish to investigate the correlation between the ES and EM waves by using time evolution of intensities. The ES waves $\left(\mathrm{L}, \mathrm{L}^{\prime}, 2 \mathrm{~L}, 3 \mathrm{~L}\right)$ represented by triangles, diamonds, rectangles, and stars are shown in Figure 5(a), and EM waves $(2 \mathrm{H}, 3 \mathrm{H}, 4 \mathrm{H})$ represented by triangles, stars, and diamonds are shown in Figure 5(b). The intensity of ES waves, except $\mathrm{L}^{\prime}$, does not change once the linear saturation at the $\sim 100 \omega_{\mathrm{pe}} t$ has taken place. The almost simultaneous excitation of ES harmonics, as shown in Figure 5(a), is still somewhat of a mystery, despite Gaelzer et al.'s (2003) explanation. The intensity of $\mathrm{L}^{\prime}$, which results from the nonlinear process, gradually increases during entire simulation time period. These findings are consistent with earlier works (Klimas 1983; Kasaba et al. 2001).

EM harmonics show entirely different dynamical behavior when compared to ES harmonics. Figure 5(b) shows that the intensity of $2 \mathrm{H}$ keeps increasing much beyond the L-mode 
saturation. When the intensity of the $2 \mathrm{H}$ mode reaches $\sim 10^{-7}$, it can be seen that the intensity associated with the $3 \mathrm{H}$ mode begins to increase. Subsequently, $4 \mathrm{H}$ mode intensity begins to be enhanced when the $3 \mathrm{H}$ mode intensity reaches $\sim 10^{-8}$. This sequential excitation of higher-harmonic EM radiation when the adjacent lower harmonic mode has reached a threshold is nicely explained by the Zlotnik-Cairns process, and is typical of nonlinear wave-wave dynamics. It is rather clear that the excitation of the $2 \mathrm{H}$ mode is intimately related to the excitation of $\mathrm{L}^{\prime}$ mode, the excitation of $3 \mathrm{H}$ depends on the $2 \mathrm{H}$ mode exceeding threshold intensity, $4 \mathrm{H}$ on $3 \mathrm{H}$ intensity, and so forth. As the generation of $2 \mathrm{H}$ is a result of $\mathrm{L}+\mathrm{L}^{\prime}$ coalescence, it is no surprise that the $2 \mathrm{H}$ intensity follows the increase in the $\mathrm{L}^{\prime}$ mode intensity. In the same vein, the $3 \mathrm{H}$ mode is generated via the coupling $\mathrm{L}+2 \mathrm{H} \rightarrow 3 \mathrm{H}$, the $4 \mathrm{H}$ mode via $\mathrm{L}+3 \mathrm{H} \rightarrow 4 \mathrm{H}$, and so forth. This is the essence of the Zlotnik-Cairns process.

Yi et al.'s theory, on the other hand, does not explain this type of sequential dynamic progression of harmonics generation. Their theory (which is essentially similar to that proposed by Gaelzer et al. 2003) does, however, explain why $2 \mathrm{~L}, 3 \mathrm{~L}, \ldots$ should saturate simultaneously with the L mode. In this regard, Yi et al.'s mechanism explains the result shown in Figure 5(a). However, one of the consequences of their theory is that multiple harmonics of the EM mode should also behave in a similar fashion dynamically, which is of course, at variance with the present simulation (Figure 5(b)). Therefore, we conclude that as far as the multiple harmonic EM emission is concerned, the Zlotnik-Cairns process is a more likely explanation.

\section{CONCLUSIONS}

The radiation emission at higher harmonics of plasma frequency has been observed in solar and interplanetary environment (Kliem et al. 1992; Zlotnik et al. 1998; Takakura \& Yousef 1974; Benz 1973; Cairns 1986). However, the issue of whether such a phenomenon is even possible in plasmas has not been resolved. In the present paper, we have thus performed two-dimensional EM full PIC numerical simulation in order to address the issue of multiple harmonic plasma emission. We have demonstrated that multiple harmonic EM emission from fundamental to $5 \mathrm{H}$ is indeed possible. Such a finding has not been reported in the literature, although similar simulations have been performed in the past. The previous simulations, however, were only concerned with EM emission at the fundamental and 2H (Pritchett \& Dawson 1983; Yin et al. 1998; Kasaba et al. 2001). In contrast, in the present paper we have shown that multiple harmonic EM emission is indeed possible. Our simulation also confirmed the excitation of ES harmonics that have been discussed before by means of simulations (e.g., Klimas 1983; Kasaba et al. 2001) and theory (e.g., Yoon et al. 2003).

We have tested and verified the competing mechanisms for multiple harmonic EM emission on the basis of the present simulation results. These include the mechanism proposed by Zlotnik (1978) and generalized by Cairns (1988), and an alternative model by Yi et al. (2007). From the detailed analysis, we conclude that, while the theory by $\mathrm{Yi}$ et al. adequately explains the excitation of ES multiple harmonic modes, as far as the EM multiple harmonic emissions are concerned, it is the Zlotnik-Cairns theory that better explains the simulation results. Specifically, we found that the Zlotnik-Cairns model provides qualitative explanations for the radiation-beam pattern and dynamical evolution. In contrast, Yi et al.'s mechanism provides a consistent picture for the multiple harmonic ES modes, but it does not seem to offer consistent explanation for the EM harmonic components.

This research was supported by grant R01-2007-000-20196-0 from KOSEF and by StSc project from POSCO. The computer resource used in simulation was supported by grant KSC-2007G02-1001 from KISTI. P.H.Y. acknowledges AFOSR contract FA9550-07-0053.

\section{REFERENCES}

Benford, G., Tzach, D., Kato, K., \& Smith, D. F. 1980, Phys. Rev. Lett., 45, 1182

Benz, A. O. 1973, Nat. Phys. Sci., 242, 39

Cairns, I. H. 1986, J. Geophys. Res., 91, 2975

Cairns, I. H. 1987, J. Plasma Phys., 38, 199

Cairns, I. H. 1988, J. Geophys. Res., 93, 858

Gaelzer, R., Yoon, P. H., Umeda, T., Omura, Y., \& Matsumoto, H. 2003, Phys. Plasmas, 10, 373

Goldman, M. V. 1983, Sol. Phys., 89, 403

Hutchinson, I. H., Molvig, K., \& Yuen, S. Y. 1978, Phys. Rev. Lett., 40, 1091

Intrator, T., Hershkowitz, N., \& Chan, C. 1984, Phys. Fluids, 27, 527

Kasaba, Y., Matsumoto, H., \& Omura, Y. 2001, J. Geophys. Res., 106, 18693

Kliem, B., Krüger, A., \& Treumann, R. A. 1992, Sol. Phys., 140, 149

Klimas, A. J. 1983, J. Gephys. Res., 88, 9081

Matsumoto, H., \& Omura, Y. 1993, in Computer Space Plasma Physics: Simulation Techniques and Software, ed. H. Matsumoto \& Y. Omura (Tokyo: Terra Sci. Pub.)

Melrose, D. B. 1985, in Solar Radiophysics, ed. D. J. McLean \& N. R. Labrum, (New York: Cambridge Univ. Press)

Parks, G. 2003, Physics of Space Plasmas, (2nd ed.; MA: Westview Press)

Pritchett, P. L., \& Dawson, J. M. 1983, Phys. Fluids, 26, 1114

Reiner, M. J., Stone, R. G., \& Fainberg, J. 1992, ApJ, 394, 340

Robinson, P. A., \& Cairns, I. H. 1998a, Sol. Phys., 181, 363

Robinson, P. A., \& Cairns, I. H. 1998b, Sol. Phys., 181, 395

Robinson, P. A., \& Cairns, I. H. 1998c, Sol. Phys., 181, 429

Takakura, T., \& Yousef, S. 1974, Sol. Phys., 36, 451

Whelan, D. A., \& Stenzel, R. L. 1985a, Phys. Rev. Lett., 47, 95

Whelan, D. A., \& Stenzel, R. L. 1985b, Phys. Fluids, 28, 958

Willes, A. J., Robinson, P. A., \& Melrose, D. B. 1996, Phys. Plasmas, 3, 149

Yi, S., Yoon, P. H., \& Ryu, C.-M. 2007, Phys. Plasmas, 14, 013301

Yin, L., Ashour-Abdalla, M., El-Alaoui, M., Bosqued, J. M., \& Bougeret, J. L. 1998, J. Geophys. Res., 103, 29619

Yoon, P. H. 1995, Phys. Plasmas, 2, 537

Yoon, P. H. 2006, Phys. Plasmas, 13, 022302

Yoon, P. H., Gaelzer, R., Umeda, T., Omura, Y., \& Matsumoto, H. 2003, Phys. Plasmas, 10, 364

Yoon, P. H., Yi, S., \& Ryu, C.-M. 2005, Phys. Plasmas, 12, 052305

Ziebell, L. F., Gaelzer, R., \& Yoon, P. H. 2001, Phys. Plasmas, 8, 3982

Zlotnik, E. Ya. 1978, Sov. Astron., 22, 228

Zlotnik, E. Ya., Klassen, A., Klein, K.-L., \& Mann, G. 1998, A\&A, 331, 1087 